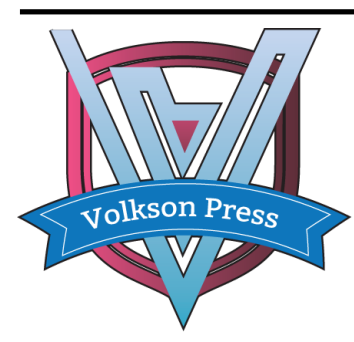

Contents List available at VOLKSON PRESS

New Materials and Intelligent Manufacturing (NMIM) DOI : http://doi.org/10.26480/icnmim.01.2018.146.148

Journal Homepage: https://topicsonchemeng.org.my/

ISBN: 978-1-948012-12-6

\title{
MULTIFUNCTIONAL XY CURVE DRAWING OF FUNCTION TREND CONTROL BASED ON WINCC
}

\author{
Tong Shaowei, Chen Zhimin, Wang Xin, Zhang Xiaodong* \\ School of information and control engineering, Jilin Institute of Chemical Technology Chengde Street, Jilin, China. \\ *Corresponding Author Email: 891642148@qq.com
}

This is an open access article distributed under the Creative Commons Attribution License, which permits unrestricted use, distribution, and reproduction in any medium, provided the original work is properly cited

\section{ARTICLE DETAILS}

Article History:

Received 26 June 2018 Accepted 2 July 2018 Available online 1 August 2018

\section{ABSTRACT}

Using C script language to process data and control variables of WINCC function trend control, a multi-function XY curve drawing based on WINCC function trend control is implemented. This method creates multiple custom array variables in the WINCC global C script, stores the collected data in the corresponding array variables, uses $C$ script language to process the stored data, and then draws the function trend control according to the relationship between variables, and completes the rendering of the multi-function XY curve. The drawn curve overcomes problems such as missing, overlapping, trailing, and unmaintainable curves caused by time changes or switching of the operation screen in the WINCC function trend control. Finally, data acquisition is triggered according to whether time or data changes, so as to ensure the real-time data acquisition.

\section{KEYWORDS}

WINCC function trend control, XY curve, C script, data acquisition.

\section{INTRODUCTION}

The WINCC function trend control has strong functions, which can meet the needs of most users in drawing process curves. Usually, the curve drawing is connected directly with the data, and the method mentioned in this paper is to create an array in the WINCC global C script, to store the detected variable data in the array, and to read the data in the array in real time by the scripting language and then draw the XY curve. The advantage of this method is that the curve can be drawn and the data can be recorded and processed at the same time. This can effectively avoid the occurrence of the loss of the original curve, trailing, overlapping and unable to maintain due to the switching of the WINCC project. It is convenient for the operator to monitor and analyze the real-time XY curve [1].

\section{DRAWING OF MULTIFUNCTIONAL XY CURVE}

Since the drawing data of the multi-functional XY curve are derived from the array in the global script, "none" is selected in the "provider" of the "data connection mode" of the WINCC function trend control. This means that the data of drawing curves do not need to come from online variables, archiving variables or user archiving, but the measured variable data are directly stored in a custom array. The number of the array is the same as the number of the acquisition variables, and the curve data is directly invoked from the corresponding array when the user draws the curve. Data acquisition is triggered by time or data changes. When a data is collected, the $\mathrm{C}$ script language will draw the data directly into a function trend control to ensure the real-time rendering of the data [2].

The author introduces the multi-function $X Y$ curve of the water content and temperature of the vertical section of the setting tank in the oil field combined station as an example:

According to the production requirements, the change of water content and temperature of different height in the setting tank can be detected, and the multi-function XY curve based on the high position moisture content and temperature is drawn on the same trend map. The position data is collected by the absolute encoder installed on the stepping motor, and the temperature and moisture content data are from the sensing detection mechanism dragged by the motor. The sensor detection mechanism of the motor is moving in the way of uplink detection or in the way of downlink detection in the tank. Then the encoder is used to record the location of the mechanism in real time, and the detection mechanism needs to measure the temperature and moisture content of the current position,store the acquisition information in the custom array.

The effective data of the uplink movement are extracted by the C script language, and the trailing, overlapping and invalid downlink data in the array are removed, and the corresponding multifunction $\mathrm{XY}$ curve is plotted according to the variable relation transfer to the function trend control. The height of the crude oil settling tank is 10.4 meters. The measurement of water content and temperature in the tank can be completed for 20 minutes. The temperature and water content in different detection positions are recorded at intervals of per second. Taking the position of the detection mechanism of the sensor in the tank as the vertical coordinate, the crude oil temperature in the tank and the water content in the tank are abscissa, and the three sets of data are displayed in a function trend control. The displayed results are retained after the screen is switched to ensure the integrity of the drawing curve. The curve is empty and waiting for the next curve to be drawn only when the artificial setting is cleared [3]. The drawing of multi-functional XY curves can be divided into three parts: data acquisition and storage, data processing and curve drawing, and redrawing of the screen switching curve.

\subsection{Data acquisition and storage}

Because the drawing data of the multi-function XY curve comes from the array, first define the array and initialize the array data, that is, the initial value of the array is zero. According to the work requirement of the detection system, the longest time of acquisition is 20 minutes. If the data is collected once per second, the length of the array is only more than 1200 floating points. (the length of the array defined by this drawing is 2000 floating points, far reaching the definition requirement). Secondly, the three sets of data detected in the process of the system upstream detection 
are collected and stored in the corresponding array according to the sequence of system work requirements, so as to realize the real-time storage of the collected data [4]. The specific introduction of the program is shown in Figure 1.

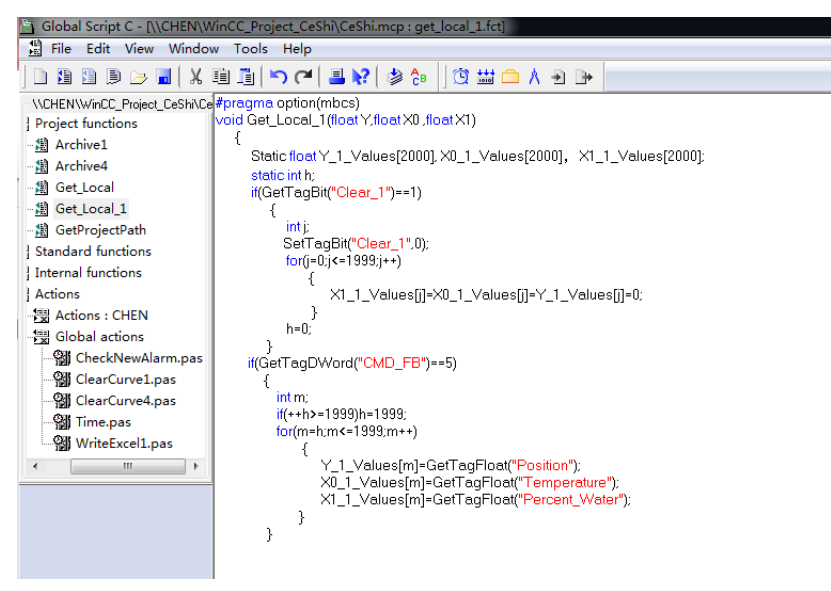

Figure 1: Data acquisition of C script

The script language is created in the standard function of the WINCC global C script to create a custom function named "Get_Local_1". Create a custom array and variable within this function, when the user defines a clean - up process (that is, Clear_1 equals 1), clearing the data and waiting for the next data collection. When the system performs uplink detection (i.e., when CMD_FB equals 5, data acquisition is started), the data is collected and stored in the corresponding array.

\subsection{Data processing and curve drawing}

After completing the collection and storage of data, 3 groups of data collected in real time are obtained and stored in the corresponding array in sequence, then the call of the data and the drawing of the curves are carried out. The steps of data processing and curve drawing are as follows:

First, create two new graphic in the WINCC graphic editor, insert the function trend control and two buttons in the first graphic, and define the name of the function trend control (such as "Trend1"). Because script language is used to edit controls, there is no need to control data connection and other object connection settings, as shown in Figure 2. The purpose of adding two buttons is to realize zero processing (that is, when the button moves, the variable "Clear_1" produces 1 actions) and the drawing switch in the process of curve drawing.Add a button to the second graphic. The aim is to switch to the graphic and click the button to switch back to the original drawing curve. This verifies that the control function written in the $\mathrm{C}$ scripting language can switch back to the original curve trend graphic when the graphic is switched, while the curve can still be drawn, and the existing curve can not be lost.

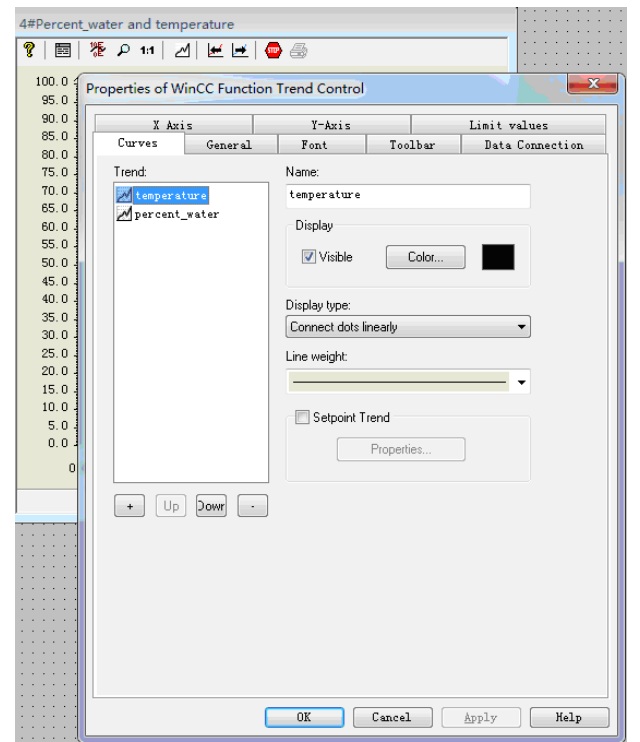

Figure 2: New function trend control
The data processing program was originally written with the $\mathrm{C}$ script. The process includes the following steps: (1)the initialization of a function trend control. The initialization includes the definition of the index name of the specific curve,the definition of the interval of the curvilinear axis, whether to display the ruler, the color of the curve, and other user's custom Settings. (2) The real-time update of the data and the drawing of the curve. Under the premise that the screen is not switched, the user has no variable connection in the control process using the function trend control, so the data collection in the control is not from the connection with the variable, but the array is used to assign the value. In this way, the connection between the array and the control is established, thus the realtime updating of the data and the drawing of the curve are realized, and the effect of the function trend control connected by direct variables is the same as that of the drawn curve.

The implementation code of the C script is shown below (the following code is tested on the two versions of WinCC 6.2 SP2 version and WinCC 7 SP3).

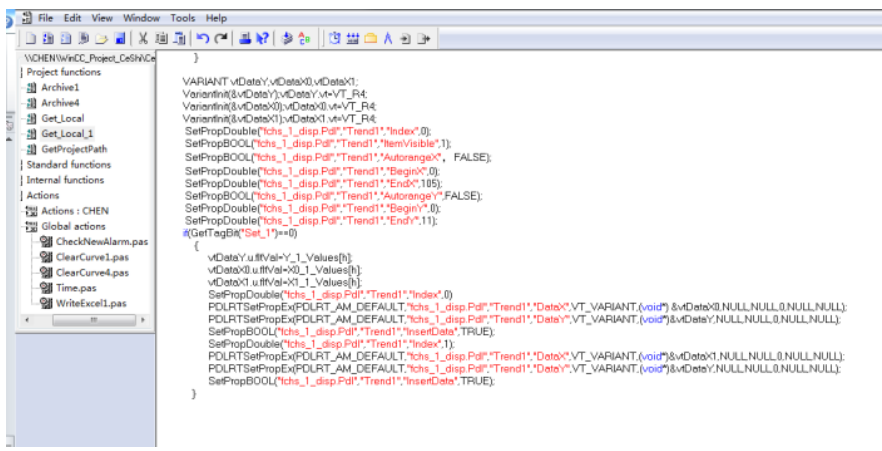

Figure 3: The curve drawing function of the scripting language

The function defines the index of the curve (which is the curve) and the interval definition (that is, the ordinate range and the abscissa range). When drawing the curve without changing the picture, the two sets of data are plotted in different curvilinear indexes respectively, so as to realize the requirement of drawing two curves at the same time.

\subsection{Redrawing of the curve after switching the graphic}

In the WINCC function trend control, there is such a problem that when other graphics switch to the original control graphic, the curve data will be lost, trailing and overlapping [5]. so after switching the graphic, the data in the original trend control should be cleared up first. Then the array data is recalled to draw the curve, and the redrawing of the curve after the switch is completed. This ensures the restoration of lost data and the realtime rendering of curves. The function is shown in Figure 4.

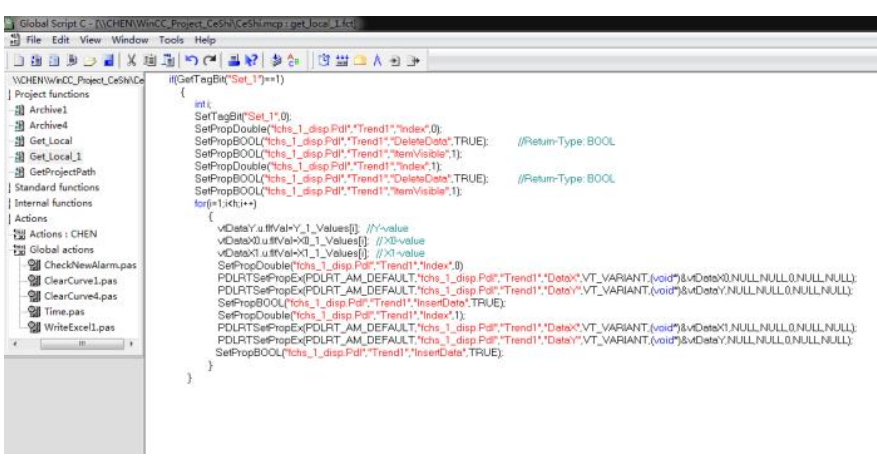

Figure 4: The drawing function of the switch screen

The function is to clean the data of the trend control data and reassign the original array data to the function trend control when the other pictures are switched to the curve trend control screen. After drawing the collected data, the curve is stopped and the data is retained in the array, and the original curve can be resumed after the next switch [6].

After the completion of the above program, because the project function is written completely in the WINCC global C script, the cycle of the function 
is one second, so it is necessary to set the sampling time of the system. That is, to set up a clocked flip-flop with per second cycle in the action [7].

\section{THE DRAWING EFFECT OF MULTIFUNCTION XY CURVE}

The actual results of the secondary settling tank in the oil field plotted by the WINCC multi-function XY curve are shown in Figure 5. The two curves are the XY curve of longitudinal position and moisture content(red curve), $\mathrm{XY}$ curve of longitudinal position and temperature (black curve). The curve drawing begins from the origin as the starting zero point. With the change of the location of the sensor in the tank, the collected data information is gradually added to the array and is drawn in the WINCC function trend control until the detection mechanism reaches a specified position [8]. Using this method, the curve of variable data is drawn. After switching the graphic, the curve can still keep the running state before switching and continue drawing until the complete curve is drawn. This method of drawing curves is convenient for operators to monitor, collect and analyze the water content data of crude oil in the settlement tank of the oil field combined station.

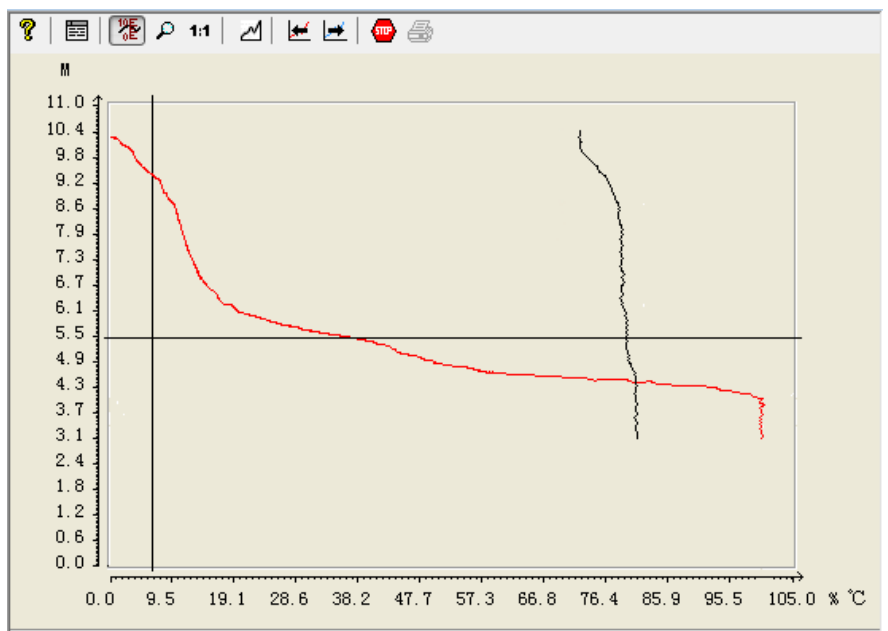

Figure 5: Water content and temperature curve of settling tank

\section{CONCLUSION}

The WINCC function trend control and the $\mathrm{C}$ script programming language combine to realize the multifunction XY curve drawing, and effectively overcome the trailing, overlap and loss of the curve caused by the traditional single variable connection.Users can consult the similar method to deal with the collected data according to actual needs, so as to draw a custom curve that meets the requirements. Practice shows that this method is simple and practical, real-time and effective, and convenient for users.

\section{REFERENCES}

[1] Zhan, Z.H. 2002. The application of Siemens S7-300 and industrial control configuration software WinCC. Science \& Technology of Baotou Steel Corporation, 22-27.

[2] Liu, X.M., Wang, S. 2004. Head first Siemens WinCC V6. Beijing, China: Beijing University of Aeronautics and Astronautics Press.

[3] Wen, Y.R. 2005. Use API function to improve the curve velocity in VB. Journal of chongqing technology and business university, 22 (2) ,148-152.

[4] Xu, Z.J. 2005. Industrial control configuration software and application. Beijing, China: Machine Industry Press.

[5] Liang, M.X. 2009. WinCC foundation and application development guide. Beijing, China: Machine Industry Press.

[6] Ma, G.H. 2001. Monitoring configuration software and its application.Beijing,China: Tsinghua University Press.

[7] Chen, X.D., Liao, C.C. 2008. Siemens man-machine interface configuration and application technology. Beijing,China: Machine Industry Press.

[8] Zhu, J.J. 2014. Monitoring configuration software WinCC application course.Beijing,China: China Electric Power Press.

\section{ABOUT THE AUTHORS}

Tong Shaowei(1963-): Graduated from the automation of production process of Jilin Institute of Chemical Technology in 1985, and taught in the same year. He has been engaged in the scientific research, engineering practice of measurement and control technology and instruments , mainly researching on industrial automatic control.

Chen Zhimin(1992-):Male, Postgraduate, Graduated from Jilin Institute of Chemical Technology. Now studying in the control engineering major of Jilin Institute of Chemical Technology. The main research direction is the integration of advanced control algorithm research and control system.

Wang Xin(1994-):Graduated from Jilin agricultural science and technology institute.Master of control engineering of grade 2017 of jilin chemical engineering college. The main research direction is the integration of advanced control algorithm research and control system.

Zhang Xiaodong (1965-

): Female, Siping City, Jilin Province, received master degree from Dalian University of Technology in 2005, associate professor of Jilin Institute of Chemical Technology, mainly researching on pattern recognition and ima ge processing. 